\title{
Electron acoustic solitary waves with non-thermal distribution of electrons
}

\author{
S. V. Singh ${ }^{1,2}$ and G. S. Lakhina ${ }^{1}$ \\ ${ }^{1}$ Indian Institute of Geomagnetism Plot No. 5, Sector-18, New Panvel (W), Navi Mumbai-410218, India \\ ${ }^{2}$ Radio Science Center for Space and Atmosphere (RASC), Kyoto University Gokasho, Uji, Kyoto 611-0011, Japan
}

Received: 3 August 2003 - Revised: 3 February 2004 - Accepted: 4 February 2004 - Published: 14 April 2004

Part of Special Issue "International Workshops on Nonlinear Waves and Chaos in Space Plasmas"

\begin{abstract}
Electron-acoustic solitary waves are studied in an unmagnetized plasma consisting of non-thermally distributed electrons, fluid cold electrons and ions. The Sagdeev pseudo-potential technique is used to carry out the analysis. The presence of non-thermal electrons modifies the parametric region where electron acoustic solitons can exist. For parameters representative of auroral zone field lines, the electron acoustic solitons do not exist when either $\alpha>0.225$ or $T_{c} / T_{h}>0.142$, where $\alpha$ is the fractional non-thermal electron density, and $T_{c}\left(T_{h}\right)$ represents the temperature of cold (hot) electrons. Further, for these parameters, the simple model predicts negatively charged potential structures. Inclusion of an electron beam in the model may provide the positive potential solitary structures.
\end{abstract}

\section{Introduction}

Bursts of broadband electrostatic noise (BEN) emissions have been observed in auroral and other regions of the magnetosphere, e.g. polar cusp, plasma sheet boundary layer (PSBL). Frequencies of these emissions range from ion plasma and lower hybrid frequencies to electron plasma frequency and above. However, most of the power is concentrated in the vicinity of ion plasma and lower hybrid frequencies. Ion and electron beams are also commonly observed during the BEN events. The generation of low frequency part of BEN can be explained by the linear theory of ion and electron-beam instabilities such as, ion- and electronacoustic instabilities. However, no linear electrostatic wave mode has been identified in the frequency range between electron plasma and cyclotron frequencies i.e. to explain the high frequency part of the BEN.

High time resolution observations have shown that the BEN consists of small scale, large amplitude magnetic field aligned electric field structures. Electric field amplitude can reach up to $100 \mathrm{mV} / \mathrm{m}$ in dayside auroral zone and more in

Correspondence to: S. V. Singh

(satyavir@iigs.iigm.res.in) auroral kilometric radiation(AKR) source region. Such large value of electric fields suggest that the nonlinear effects have a bearing in the generation of BEN. Electrostatic solitary structures have been observed in the auroral acceleration region (Temerin et al., 1982; Bostrom et al., 1988; Dubouloz et al., 1991a, b; Mozer et al., 1997; Ergun et al., 1998; Bounds et al., 1999), in Earth's high altitude polar magnetosphere (Franz et al., 1998), in Polar cap boundary layer (Tsurutani et al., 1998), in the plasma sheet boundary layer (Matsumoto et al., 1994; Omura et al., 1994), on cusp field lines (Cattell et al., 1999) and in AKR source region (Pottelette et al., 1999).

These small scale, large amplitude electrostatic spiky structures are interpreted as solitary waves with velocities from few hundred to few thousand $\mathrm{km} / \mathrm{s}$ and parallel scale sizes 100-1000 m. Dubouloz et al. (1991) studied the electron-acoustic solitons in unmagnetized plasma with two electron-component (cold and hot) and motionless ions. It was shown that the high frequency extension of BEN in dayside auroral zone could be generated by the electronacoustic solitons passing by the satellite. Later on above study was extended to magnetized plasma to explain the turbulence generated by the gas of electron-acoustic solitons (Dubouloz et al., 1993) in dayside auroral zone. Mace et al. (1991) studied the arbitrary amplitude electron-acoustic solitons in a two electron component plasma. The above mentioned models are unable to explain the positive polarity of observed solitary structures. Berthomier et al. (2000) and Singh et al. (2001a) have studied the electron-acoustic solitons in four-component plasma. Berthomier et al. (2000) showed that, depending upon the beam velocity, temperature and density, electron-acoustic solitons can have positive polarity. Mamun et al. (2002) have studied the obliquely propagating electron-acoustic solitary waves in a magnetized plasma. Lakhina et al. (2003) presented a review of some theoretical models for solitary structures of the boundary layer waves. Recently, Berthomier et al. (2003) showed that the small amplitude Debye length scale positive potential structures described as electron-acoustic beam solitons can form in the auroral plasma at FAST and POLAR altitudes. 
Energetic electrons distributions are also observed in the different regions of the magnetosphere. Cairns et al. (1995) used non-thermal distribution of electrons to study the ionacoustic solitary structures observed by the FREJA satellite. It was shown that solitons with both positive and negative density perturbations could exist. We study the effect of non-thermal electron distribution on the nonlinear electron-acoustic waves in an unmagnetized three component plasma consisting of non-thermal electrons, cold electrons and ions. Our choice of non-thermal distribution of electrons is prompted by its convenience rather than as precise fitting of the observations. We expect that the inclusion of the non-thermal electrons will change the properties as well as the regime of existence of solitons.

\section{Formulation}

We consider a homogeneous, unmagnetized three component plasma consisting of non-thermal hot electrons, fluid cold electrons and ions. The non-thermal distribution for the electrons is given by (Cairns et al., 1995)

$$
f_{0 h}(v)=\frac{n_{o h}}{\sqrt{2 \pi v_{t h}^{2}}} \frac{\left(1+\frac{\alpha v^{4}}{v_{t h}^{4}}\right)}{(1+3 \alpha)} \exp \left(-\frac{v^{2}}{2 v_{t h}^{2}}\right)
$$

where $n_{o h}$ is the hot electron density, $v_{t h}$ is the thermal speed of the hot electrons and $\alpha$ is a parameter which determines the population of energetic non-thermal electrons. The distribution of electrons in the presence of non-zero potential can be found by replacing $v^{2} / v_{t h}^{2}$ by $v^{2} / v_{t h}^{2}-2 \phi$. Thus, integration over the resulting distribution function gives the following expression for the electron density (Cairns et al., 1995)

$n_{h}=n_{o h}\left(1-\beta \phi+\beta \phi^{2}\right) \exp (\phi)$, and the other governing equations of the model are given by

$$
\begin{aligned}
& \frac{\partial n_{j}}{\partial t}+\frac{\partial}{\partial x}\left(n_{j} v_{j}\right)=0, \\
& \frac{\partial v_{j}}{\partial t}+v_{j} \frac{\partial v_{j}}{\partial x}+\frac{1}{\mu_{j} n_{j}} \frac{\partial P_{j}}{\partial x}-\frac{Z_{j}}{\mu_{j}} \frac{\partial \phi}{\partial x}=0, \\
& \frac{\partial P_{j}}{\partial t}+v_{j} \frac{\partial P_{j}}{\partial x}+3 P_{j} \frac{\partial v_{j}}{\partial x}=0 \\
& \frac{\partial^{2} \phi}{\partial x^{2}}=n_{h}+n_{c}-n_{i}
\end{aligned}
$$

where $j=c, i$ represents cold electrons and ions, respectively, $Z_{j}= \pm 1$ for electrons and ions respectively, and $\mu_{j}=m_{j} / m_{e}, \beta=4 \alpha /(1+3 \alpha)$, where $\alpha$ determines the population of non-thermal electrons. We have normalized the densities by $n_{o}=n_{o c}+n_{o h}=n_{o i}$, velocities by thermal velocity of hot electrons, $v_{t h}=\sqrt{T_{h} / m_{e}}$, lengths by effective hot electron Debye length defined as $\lambda_{d h}=\sqrt{T_{h} / 4 \pi n_{o} e^{2}}$, temperature by hot electron temperature $T_{h}$, time by inverse of electron plasma frequency $\omega_{p e}^{-1}=\sqrt{m_{e} / 4 \pi n_{o} e^{2}}$, the potential by $T_{h} / e$, and, the thermal pressure by $n_{o} T_{h}$.

The stationary solution to Eqs. (2-6) can be found by using the transformation $\xi=x-M t ; M$ is the velocity of the moving frame, and applying the appropriate boundary conditions. The integration of the Poisson Eq. (6) yield the energy integral given by

$$
\frac{1}{2}\left(\frac{d \phi}{d \xi}\right)^{2}+V(\phi)=0
$$

where $V(\phi)$ is the Sagdeev potential given by

$$
\begin{aligned}
V(\phi) & =n_{o h}\left\{1+3 \beta-\left(1+3 \beta-3 \beta \phi+\beta \phi^{2}\right) e^{\phi}\right\} \\
& +n_{o c}\left[M^{2}-\frac{M}{\sqrt{2}}\left\{M^{2}+3 T_{c}+2 \phi+\sqrt{\left(M^{2}+3 T_{c}+2 \phi\right)^{2}-12 T_{c} M^{2}}\right\}^{1 / 2}\right] \\
& +n_{o c} T_{c}\left[1-2 \sqrt{2} M^{3}\left\{M^{2}+3 T_{c}+2 \phi+\sqrt{\left(M^{2}+3 T_{c}+2 \phi\right)^{2}-12 T_{c} M^{2}}\right\}^{-3 / 2}\right] \\
& +\mu_{i}\left[M^{2}-\frac{M}{\sqrt{2}}\left\{M^{2}+\frac{3 T_{i}}{\mu_{i}}-\frac{2 \phi}{\mu_{i}}+\sqrt{\left(M^{2}+\frac{3 T_{i}}{\mu_{i}}-\frac{2 \phi}{\mu_{i}}\right)^{2}-\frac{12 T_{i} M^{2}}{\mu_{i}}}\right\}^{1 / 2}\right] \\
& +T_{i}\left[1-2 \sqrt{2} M^{3}\left\{M^{2}+\frac{3 T_{i}}{\mu_{i}}-\frac{2 \phi}{\mu_{i}}+\sqrt{\left(M^{2}+\frac{3 T_{i}}{\mu_{i}}-\frac{2 \phi}{\mu_{i}}\right)^{2}-\frac{12 T_{i} M^{2}}{\mu_{i}}}\right\}^{-3 / 2}\right],
\end{aligned}
$$

where $\mu_{i}=m_{i} / m_{e}$. The first term on the right hand side (r.h.s.) of Eq. (8) represents the hot electron contribution to the Sagdeev potential. In the absence of non-thermal elec- trons, i.e. for $\alpha=\beta=0$, the term reduces to usual Boltzmann distributed hot electron contribution (Mace et al., 1991; Singh et al., 2001a). The second and third term on the r.h.s. 


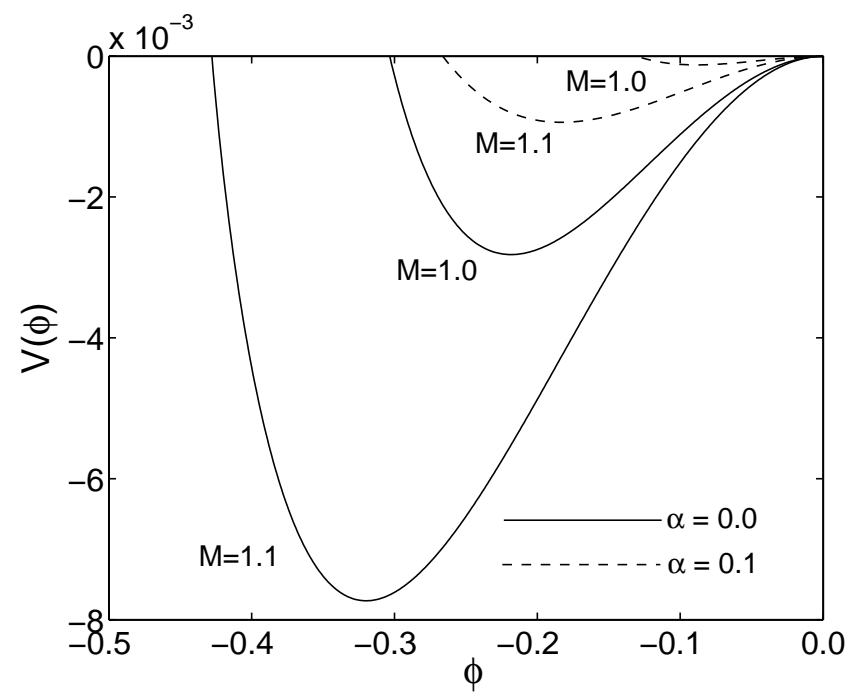

Fig. 1. Figure 1 shows the comparison of the Sagdeev potential profiles for the Maxwellian $(\alpha=0)$ and non-thermally $(\alpha=0.1)$ distributed electrons for different values of the Mach number $M$. Other parameters are $n_{o c}=0.35, n_{o h}=0.65, T_{c} / T_{h}=0.01=T_{i} / T_{h}$.

of Eq. (8) represents the cold electron contribution. The last two terms on the r.h.s. of Eq. (8) give the contribution of ions to the Sagdeev potential.

\section{Numerical results}

The soliton solutions of Eq. (7) exist when the usual conditions, namely, $V(\phi)=0, d V(\phi) / d \phi=0$ at $\phi=0$ and $V(\phi)<0$ for $0<|\phi|<\left|\phi_{o}\right|$, where $\phi_{o}$ is the maximum amplitude of the solitons, are satisfied. Equations (7) and (8) are solved numerically for the soliton amplitude and Sagdeev potential, respectively. The typical parameters considered for the numerical evaluation are: cold electron density, $n_{o c}$ $=0.35$, hot electron density, $n_{o h}=0.65$, cold to hot electron temperature ratio, $T_{c} / T_{h}=0.01=T_{i} / T_{h}$, and $\alpha=0.1$

Figure 1 shows the variation of Sagdeev potential $V(\phi)$ with normalized potential $\phi$ for the above mentioned parameters for various values of soliton Mach number $M$ indicated on the curves. It is interesting to point out that for Maxwellian electrons $(\alpha=0)$, the numerical results show that soliton solution exists for $0.76<M<1.106$. However, for $\alpha=0.1$, soliton solutions exist for $0.9<M<1.525$ and for $\alpha=0.2$, the range of Mach numbers for which soliton solution exists is $1.07<M<2.172$ for the above mentioned parameters. This implies that the Mach number regime in which soltions can exist gets modified with the inclusion of non-thermal electron distribution. In the presence of nonthermal electron population, the soliton can exist for larger soliton speeds or Mach numbers.

Figure 2 shows the comparison of the soliton profiles for the Maxwellian $(\alpha=0)$ and non-thermally (finite $\alpha$ ) distributed electrons for Mach number, $M=1.1$ and cold to hot electron temperature ratio, $T_{c} / T_{h}=0.01$. It can be seen from

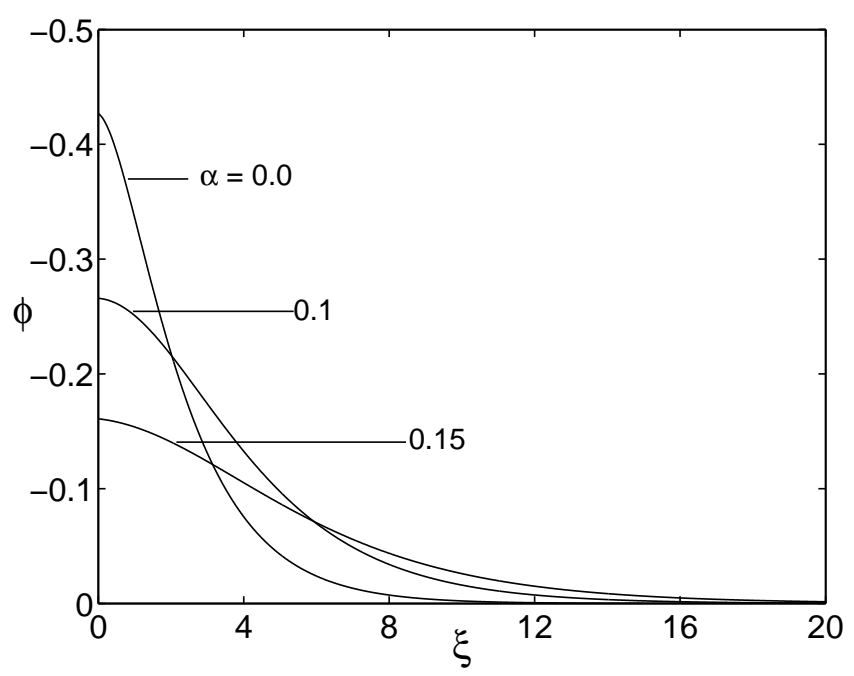

Fig. 2. Figure 2 shows the comparison of soliton profiles for the Maxwellian $(\alpha=0)$ and non-thermally $(\alpha=0.1,0.15)$ distributed electrons for $M=1.1$. Other parameters are the same as in Fig. 1.

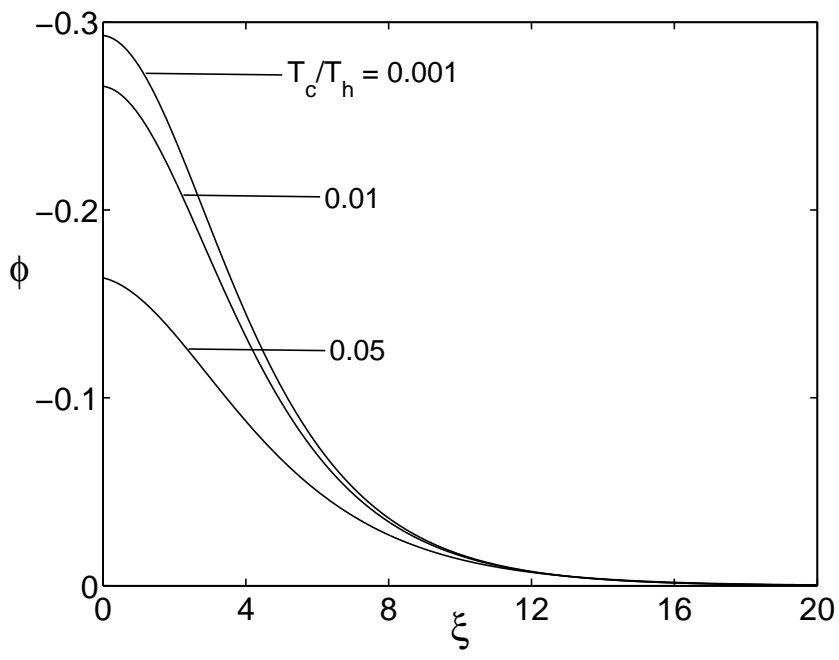

Fig. 3. Figure 3 shows the variation of soliton profiles for various values of $T_{c} / T_{h}$ as shown on the curves for $M=1.1$ and $\alpha=0.1$. Other parameters are same as in Fig. 1.

the figure that amplitude of the solitons decreases as the population of the non-thermally distributed electrons increases. For the parameters of Fig. 2, soliton solutions do not exist beyond an upper limit of $\alpha=0.225$.

Figure 3 shows the variation of soliton profiles for the non-thermally distributed electrons for different values of $T_{c} / T_{h}$ as shown on the curves for $\alpha=0.1$ and Mach number, $M=1.1$. The amplitude of the solitons decreases with the increase in $T_{c} / T_{h}$ ratio and there exist an upper limit of $T_{c} / T_{h}=0.142$ beyond which the soliton solutions are not found. 


\section{Discussion}

The choice of non-thermal distribution for energetic electrons used in our model is due to its convenience rather than as a precise fitting model of what is observed. It must be emphasized here that the inclusion of non-thermal electrons leads to the reduction in amplitude of the soliton and increase in the range of Mach numbers, for which the soliton solution exists. The electron acoustic solitons do not exist for when either $\alpha>0.225$ or $T_{c} / T_{h}>0.142$ for the parameters considered here.

For the parameters representative of auroral zone conditions, we obtain only negative electron-acoustic solitary potential structures in an unmagnetized plasma consisting of cold and non-thermally distributed electrons and ions. The normalized electric field amplitude, $d \phi / d \xi$ of these structures can be obtained by numerically integrating Eq. (7). The absolute value of the electric field comes out to be in the range of $2-100 \mathrm{mV} / \mathrm{m}$, while the observed amplitude can reach up to several hundred $\mathrm{mV} / \mathrm{m}$. The soliton velocities can be obtained from the range of Mach numbers for which the soliton solutions are valid and are found to be in the range of $4000-6000 \mathrm{~km} / \mathrm{s}$ corresponding to the hot electron temperature of $100 \mathrm{eV}$. From Figs. 2 and 3 the soliton width is found to be in the range of $10-150 \mathrm{~m}$. There is qualitative agreement between the predicted amplitudes, speeds and widths of the solitons and the observed solitary waves by POLAR and FAST spacecrafts. However, there is an important difference between the predictions of simple model discussed here and the observations in the sense that whereas the model predicts negatively charged potential structures, the observed solitary structures have positive potential.

Berthomier et al. (2000) have shown the existence of positive potential structures in a four component electron-beam plasma system, in small amplitude limit and in a very limited range. Therefore it is necessary to include an electron beam in the analysis to get the positive potential structures to bring the model closer to observations. This is beyond the scope of the present paper. It will be included in the further work on electron-acoustic solitary waves.

We have presented a simple 1-D model for the electron acoustic solitons. The stability of solitons in 2-D and 3-D needs to be examined. Recently, Berthomier et al. (2003) have developed a model of 3-D electrostatic structures. They have shown that in small amplitude limit, 3-D shape of the fast-moving coherent structures can be produced by nonlinear fluid model of 3-D electron-acoustic beam solitons. At FAST altitudes the model predicts spheroidal structures while at higher altitudes it predicts solitary waves elongated across the magnetic field.

Useful information about the life-time of the electronholes and ion heating is provided by the 2-D and 3-D particle simulations of the electron-beam driven instabilities(Omura et al., 1999; Goldman et al., 2000; Singh et al., 2001b). 3-D simulations of the electron-beam driven electron holes reveal that initially a large number of electron holes are formed. They continuously merge with each other and at a later time, only few electron holes are left and they decay by emitting low-frequency electrostatic whistler waves just above the lower hybrid frequency (Singh, 2003). Recently, Muschietti et al. (2002) have given a 3-D Bernstein-Greene-Kruskal (BGK) model to explain stretched solitary waves along the magnetic field lines observed in the downward current regions of the auroral zone. A full kinetic theory would be required to explain all features of the observed solitary structures.

Acknowledgement. SVS would like to thank H. Matsumoto for the hospitality at RASC, Kyoto University, Kyoto, Japan and JSPS for the financial support.

Edited by: J. Büchner

Reviewed by: two referees

\section{References}

Berthomier, M., Pottellette, R., Malingre, M., and Khotyaintsev, Y.: Electron-acoustic solitons in an electron-beam plasma system, Phys. Plasmas, 7, 2987, 2000.

Berthomier, M., Pottelette, R., Muschietti, L., Roth, I., and Carlson, C. W.: Scaling of 3-D solitary waves observed by FAST and POLAR, Geophys. Res. Lett., 30, 2148, 2003.

Bostrom, R., Gustafsson, G., Holback, B., Holmgren, G., Koskinen, H., et al.: Characteristics of solitary waves and weak double layers in the magnetospheric plasma, Phys. Rev. Lett., 61, 82, 1982.

Bounds, S. R., Pfaff, R. F., Knowlton, S. F., Mozer, F. S., Temerin, M. A., et al.: Solitary potential structures associated with ion and electron beams near $1 R_{E}$ altitude, J. Geophys. Res. 104, 28 709, 1999.

Cairns, R. A., Mamun, A. A., Bingham, R., Dendy, R., Bostrom, R., Nairns, C. M. C., and Shukla, P. K.: Electrostatic solitary structures in nonthermal plasmas, Geophys. Res. Lett., 22, 2709, 1995.

Cattell, C., Dombeck, J., Wygant, J. R., Hudson, M. K. Mozer, F. S., et al.: Comparisions of polar satellite observations of solitary wave velocities in the plasma sheet boundary and the high altitude cusp to those in the auroral zone, Geophys. Res. Lett., 26, 425, 1999.

Dubouloz, N., Pottellette, R., Malingre, M., Holmgren, G., and Lindqvist, P. A.: Detailed analysis of broadband electrostatic noise in the dayside auroral zone, J. Geophys. Res., 96, 3565, 1991a.

Dubouloz, N., Pottelette, R., Malingre, M., and Treumann, R.A.: Generation of broadband electrostatic noise by electron acoustic solitons, Geophys. Res. Lett., 18, 155, 1991b.

Dubouloz, N., Treumann, R. A., Pottelette, R., and Malingre, M.: Turbulence generated by a gas of electron acoustic solitons, J. Geophys. Res., 98, 17 415, 1993.

Ergun, R. E., Carlson, C. W., McFadden, J. P., Mozer, F. S., Delory, G. T., et al.: FAST satellite observations of large-amplitude solitary structures, Geophys. Res. Lett., 25, 2041, 1998.

Franz, J. R., Kintner, P. M., and Pickett, J. S.: Polar observation of coherent electric field structures, Geophys. Res. Lett., 25, 1277 , 1998.

Goldman, M. V., Gary, F., Newman, D. L., and Oppenheim, M.: Turbulence driven by two-stream instability in a magnetized plasma, Phys. Plasmas, 7, 1732, 2000. 
Lakhina, G. S., Tsurutani, B. T., Singh, S. V., and Reddy, R. V.: Some theoretical models for solitary structures of the boundary layer waves, Nonl. Proc. Geophys., 10, 65, 2003.

Mace, R. L., Baboolal, S., Bharuthram, R., and Hellberg, M. A.: Arbitrary amplitude electron-acoustic solitons in a two electroncomponent plasma, J. Plasma Phys., 45, 323, 1991.

Mamun, A. A., Shukla, P. K., and Stenflo, L.: Obliquely propagating electron-acoustic solitary waves, Phys. Plasmas, 9, 1474, 2002.

Matsumoto, H., Kojima, H., Miyatake, T., Omura, Y., Okada, M., et al.: Electrostatic solitary waves (ESW) in the magnetotail: BEN wave forms observed by GEOTAIL, Geophys. Res. Lett., 21, 2915, 1994.

Mozer, F. S., Ergun, R. E., Temerin, M., Cattell, C., Dombeck, J., et.al.: New features of time domain electric field structures in the auroral acceleration region, Phys. Rev. Lett., 79, 1281, 1997.

Muschietti, L., Roth, I., Carlson, C. W., and Berthomier, M.: Modeling stretched solitary waves along magnetic field lines, Nonl. Processes Geophys. 9, 101-109, 2002.

Omura, Y., Kojima, H., and Matsumoto, H.: Computer simulation of electrostatic solitary waves: a nonlinear model of broadband electrostatic noise, Geophys. Res. Lett., 21, 2923, 1994.

Omura, Y., Kojima, H., Miki, N., Mukai, T., Matsumoto, H., and Anderson, R.: Electrostatic Solitary Waves Carried by Diffused Electron Beams Observed by the Geotail Spacecraft, J. Geophys. Res., 104, 14 627, 1999.
Pottelette, R., Ergun, R. E., Truemann, R. A., Berthomier, M., Carlson, C. W., et al.: Modulated electron-acoustic waves in auroral density cavities: FAST observations, Geophys. Res. Lett., 26, 2629, 1999.

Singh, N.: Space-time evolution of electron-beam driven electron holes and their effects on the plasma, Nonl. Proc. Geophys., 10, 53, 2003.

Singh, S. V., Reddy, R. V., and Lakhina, G. S.: Broadband Electrostatic noise due to nonlinear electron-acoustic waves, Adv. Space. Res., 28, 1643-1648, 2001a.

Singh, N., Loo, S. M., Wells, B. E., and Lakhina, G. S.: Evolution of electron beam generated waves resulting into transverse ion heating and filamentation of the plasma, J. Geophys. Res., 106, 21 165, 2001b.

Temerin, M., Cerny, K., Lotko, W., and Mozer, F. S.: Observations of double layers and solitary waves in the auroral plasma, Phys. Rev. Lett., 48, 1175, 1982.

Tsurutani, B. T., Arballo, J. K., Lakhina, G. S., Ho, C. M., Buti, B., et al:: Plasma waves in the dayside polar cap boundary layer: bipolar, monopolar electric pulses and whistler mode waves, Geophys. Res. Lett., 25, 4117, 1998. 